Aus der Infektionsabteilung des Allgemeinen Krankenhauses Barmbeck in Hamburg. (Oberarzt: Prof. Reiche.)

\section{Ueber das Verhalten des Blutbildes bei Mischinfektion von Masern und Keuchhusten, sowie über einen Fall von Masern ohne Exanthem.}

\author{
Von Dr. E. Cohn, Assistent der Abteilung.
}

Auf der Keuclihustenstation des Allgemeinen Krankenhauses Barmbeck wurde Ende Februar d. J. ein Masernfall eingeschleppt. Im Anschluß daran erkrankte eine Anzahl von Kindern an Masern. Unter ihnen waren drei, die noch typische, heftige Keuchhustenanfälle aufwiesen; ein viertes Kind, das ebenfalls noch cliarakteristische Allfälle hatte, erkrankte mit allen sonstigen Zeichen von Masern, ohne dall ein Exanthem auftrat.

Diese Fälle boten Gelegenheit, den Uebergang vom Keuchhustenzun Masernblutbild näher zu studiereı. Das Keuclihustenblutbild ist von $\mathrm{Sch}$ nl eider dahin festgelegt, daß sich eine, zuweilen sehr lohe Leukozytose mit relativer Lymphozytose findet (über gleichgerichtete Untersuchungen aus unserer Abteilung wird demnächst bericitet werden), während umgekehrt die Masern Leukopenie und relative Lymphopenie aufzuweisen pflegent. Es war also festzustellen, ob bei den Pertussiskindern sich überhaupt ein Einfluls im Sinne des Masernblutbildes bemerkbar nachte, wann dieser begann, und wie lange er dauerte.

$\mathrm{Z}_{\text {uI }}$ bemerken ist, daß 2 Fälle, ein 2 Jahre und ein 10 Monate aites Kind, bei Beginn der Masern reine Keuchhustenfälle darstellten, während ein dritter Fall im Alter von 7 Montaten durch leichte bronchopneumonische Herde kompliziert war.

Bei ersten beiden Fällen machte sich am Tage des Exanthemausbruclis eine Leukopenie von 6400 bzw. 7600, trotz gleichzeitigen, sich über $39^{\circ}$ bewegenden Fiebers bemerkbar, während in den vorhergehenden Prodromaltagen die Leukozytenzahlen dem Fieber entsprachen und am Tage vor Ausbruch des Exanthems 19400 bzw. 14000 betrugen. Bei Fall 111 kam es, wolll infolge der bronchopneumonischen Herde, nicht zu einer Leukopenie, die Leukozytenzahl betrug hier bei Exanthemausbruch 17800 .

Im Blutbild nahmen bereits vom 10. Inkubationstage an bei bis dahin für Keuchhusten typischer Lymphozytose von 66,5, 67,0 und $85,5 \%$, die Polynukleären an Zalıl zıI und erreichten am Tage des Exanthemausbruchs $56,5,58,5$ und $62,0 \%$. Gleichzeitig trat eine nach Joch m an n für Masern charakteristische Verschiebung nach links anf. Im weiteren Masernverlauf wurde Fall 1 von schwerster Bronchopreumonie und Otitis media befallen, denen er späterhin erlag; das Blutbild wurde vom 5. Tage $a b$ von diesen Komplikationen beherrscht. Am 2. Tage nach Ausbruch des Exanthems stiegen die Lyınphozyten um ein Geringes, anf $50,5 \%$ an, am 3 . Tage trat eine postinfektiöse vorübergehende Eosinophilie von 10\% auf.

Bei Fall 11 zeigten sich unmittelbar nach Ausbruch des Exaithems leichte bronchopneumonische Herde, denen das Blutbild entsprach; nach deren Abklingen überwog vom 6. Tage ab der KeuchhusteneinfluB.

Bei Fall 111 war bereits am Tage nach dem Ausbruch des sehr fliichtigen, kaum 24 Stunden währenden Exanthems das Keuchhustenblutbild mit 29600 Leukozyten und 64,0\% Lymphozyten wiederhergestellt, das nur einmal durch deı Ausbruch von Varizellen an 4. Tage unterbrochen wurde.

Von besonderem Interesse ist Fall 1V. Das 12 Monate alte Kind, das mit Fall 11 und 111 in einem Zimmer lag und mit Sicherheit früher Maserı nicht gehabt hatte, litt neben einem mittelschweren Keuchhusten an spasnoplilen Krämpfen. Am selben Tage, an dem bei beiden anderen Kindern die Prodrome einsetzten, begann auch bei diesem Kind die Temperatur zu steigen, und es trat eine Konjunktivitis auf; Koplicksche Flecke, die bei den andern Kindern am 12. Inkubationstage vorhanden waren, wurden hier vermi $\beta t$, dagegen zeigte sich ein langsames Absinken der Leukozyten, die an dem Tage, an dem bei I bis 111 das Exanthem ausbrach, den Wert von 8000 erreichten, um dann wieder anzusteigen. Im Blutbild war vom 12. Inkubationstage ab ein Ansteigen der Polynukleären $\mathrm{zu}$ bemerken, das bis $51,0 \%$ ging, während die Zahl vorher $18 \%$ betragen hatte. Gleiclizeitig fand eine Verschiebung nach links derart statt, daß am 14. Inkubationstage in 100 Polynukleären 127 Kernsegmente gezählt wurden. Der Fall verlief nur einen Tag unkompliziert, an diesem Tage war ein Ansteigen der Lymphozyten auf $49 \%$ zu verzeiclmen, an folgenden Tage trat eine Otitis media später eine schwere Bronchopneumonie und Enteritis ein, denen das Kind erlag. Die Sektion (Prof. F a h r) ergab neben Bronchopneumonie, Enteritis und Otitis vor allen ein völliges Fehlen der Tliymus bel dem 12 Monate alten Kinde. Auch mikroskopisch wurden in dem an dessen Stelle befindlichen Fettgewebe Drüsenreste nicht gefunden. Epithelkörper o. B.

Der Fall bot also, abgesehen von Exanthen, alle Zeichen der Masern, einschließlich Leukozytenverminderung und Verschiebung nach links. Bei den klaren lnfektionsbedingungen dürfte kaum daran zu zweifelı seiı, daß wir diesen Fall als einen der seltenen von Morbilli sine exanthenate und olue Koplicksche Flecke erweisen können der sich hier bei einem 12 Monate alten spasmophilen Kinde mit völliger Aplasie der Thynus abspiclte. Wie selten solche Fälle sind, geht darans hervor, daß $\mathrm{Heubner}$ nur über einen einzigen, $\mathrm{Schütz}$ über zwei, $\mathrm{Salzer}$ gleichfalls über einen Fall berichten. Von diesen verlief der Salze rsclte ebenfalls letal, während $S$ ch üt $z$ und $H$ enbner bei inten drei Kindern nur leichte Erkrankungen sahen.

Schneider, M. m. W. 1914 Nr. 6. - Jochmann, Lb. d. Infektkrkh. 1914, Heubner, Lb. d. Kindhlk. 1, S. 289. - Schütz, M. m. W. 1905 S. 557 . - Salzer M. m. W. 1905 S. 360. 